\title{
Decision processing in memory: Factors influencing the storage and retrieval of linguistic and form identification*
}

\author{
STEVEN SCHWARTZ and KIRK D. WITHERSPOON \\ Northern Illinois University, DeKalb, Illinois 60115
}

\begin{abstract}
As a test of the notion that the meaning of a sentence is stored in a schematic fashion, Bransford and Franks (1971) presented the results of recognition experiments indicating that new sentences containing all of the information conveyed by simpler sentences are more likely to be misidentified as having been seen earlier than sentences containing only part of the earlier information. The present experiment, employing a signal detection framework, found this effect to be due not only to schematic organization, but to the retention of the "form" of the originally presented sentences as well. The form of new sentences conveying only part of the earlier information was better remembered (and less frequently confused) than new, complex sentences. The implications of this finding for a schematic view of memory are discussed.
\end{abstract}

Bartlett's (1932) view of memory, namely, that information is integrated into coherent organizations or schemas, formed the basis for a series of experiments conducted by Bransford and Franks (1971). In these studies, complex sentences (henceforth referred to as "Level 4," L-4, sentences because each expressed four independent ideas) were broken down into simpler constituent sentences, each expressing one (L-1), two (L-2), or three (L-3) of the original four ideas. Some of these sentences were presented during an acquisition phase and some, including the original L-4 sentences, were presented only in a recognition phase. New sentences (those appearing only in recognition) were frequently misidentified as having appeared during acquisition. In addition, Ss were most confident of having previously encountered those new sentences that contained all of the semantic information acquired during acquisition. Thus, L-4 sentences, containing four of the ideas conveyed in simpler form during acquisition, although never actually presented, were likely to be misidentified as "old" with high confidence. New sentences expressing three of the ideas (L-3s) were misidentified with somewhat less confidence, L-2s with still less confidence, and $\mathrm{L}-1$ sentences with the least confidence of all. Replications of these studies by Cofer and his associates (1973) have demonstrated the reliability of this finding.

Cofer, as well as Bransford and Franks, viewed his results as supporting the notion that memory in the recognition task is schematically organized. Semantic information, they argued, was integrated into schemas across nonsequential sentences. New sentences, then, were misrecognized as "old" when they contained the

\footnotetext{
*The authors wish to express their appreciation to D. E. Broadbent whose advice guided much of the research program underlying the present paper as well as to C. N. Cofer who read and commented on an earlier draft of the paper.
}

same information present in the schema. There is, however, another equally likely explanation for their findings. The actual (verbatim) "form" of the simpler sentences may have been easier to recall than the form of the longer, more complex sentences, thereby making them less likely to be confused with new sentences. From this view, as we proceed up the scale from L-1 to L-4 sentences, we find greater numbers of misidentifications not because of schematic organization but because the form of the sentences becomes more difficult to remember.

The present experiment was designed so as to allow a distinction between the two alternatives. If memory is organized in terms of schemas, then one would expect that, when faced with uncertainty, Ss would be inclined to respond "old" to those sentences containing the same semantic information as the schema independent of how well the form of the particular sentence was remembered. In other words, it should be possible to demonstrate different decision criteria for sentences of varying levels. If, on the other hand, misidentifications occurred more frequently and with greater confidence to the higher level sentences because they were more difficult to remember (after all, they tended to be long and syntactically complex), then memory for the exact form of the sentences should vary in an inverse manner with sentence level independent of any decision criterion. In a situation in which both the original sentences as well as new sentences are presented during recognition, an operating characteristic relating the probability of a correct response to that of an error (false alarm) can help to distinguish between the two possibilities. A change in bias toward complex sentences should produce an increase in correct responses as the false alarm rate increases, while differences in the memory for sentence form will lead to a change in the number of correct responses with a constant false alarm 
rate. In decision theory terms, memory for form will be mirrored by differences in $\mathrm{d}^{\prime}$ while schematic processes will be reflected by differences in $\beta$.

\section{METHOD}

The Ss were 15 introductory psychology students of normal vision and hearing who received course credit for participation. The experiment was conducted in the university language laboratory. The room was arranged with desks, each containing a set of monophonic earphones, separated by side panels. The room was arranged so that each $\mathrm{S}$ had a clear view of a screen on which slides con taining the various sentences were projected.

Four complex four-idea sentences taken from Bransford and Franks (1971) formed the basis for the present experiment. From each complex sentence (e.g., "The rock which rolled down the mountain crushed the tiny hut at the edge of the woods") three subtypes were formed: Level 1 (L-1) sentences containing a single, simple idea (e.g., The hut was tiny); Level 2 (L-2) sentences containing two ideas; and Level 3 (L-3) sentences containing three of the ideas. Sentences employing the connective "and" to join two or more ideas were avoided, thereby leaving for each four-idea sentence (L-4) 4 L-1s, 4 L-2s, and $3 \mathrm{~L}-3 \mathrm{~s}$ for a total of 12 sentences per L-4, or 48 sentences in all. Half of the sentences at each level (i.e., 8 L-1s, 8 L-2s, and 6 L-3s) excluding the L-4s were chosen for presentation during acquisition. All of the sentences, including those presented during acquisition as well as the $4 \mathrm{~L}-4 \mathrm{~s}$, were presented in the recognition phase. Each sentence was typed and photographed in order to produce $35-\mathrm{mm}$ black and white slides, each containing a single sentence.

\section{Procedure}

Upon entering the language laboratory, each $\mathrm{S}$ was seated at a booth, asked to put the earphones on, and given an instruction and answer booklet. Instructions were broadcast over the earphones and also appeared in the booklets. These instructions informed the Ss that the experiment was concerned with memory and that they would be seeing sentences projected at a rate of one every $5 \mathrm{sec}$. The Ss were asked to remember each of them. The sentences chosen for acquisition were presented in a random order in a darkened room in order to discourage Ss from making entries in their answer booklets. After the presentation of the acquisition sentences, Ss were informed that a second series of sentences would be presented and that for each sentence they would have to judge whether it had appeared in the original group of sentences or whether it was new. Immediately following each sentence, during the recognition phase, there was a 5-sec period of illumination during which the Ss were required to indicate their judgment on a 5-point scale ranging from 1 ("certain this sentence did not appear in the original group") to 5 ("certain this sentence did appear in the original group"). The rating scales were printed in the instruction and answer booklets. The presentation of sentences during the recognition phase was pseudorandom, arranged so that two sentences formed from the same L-4 never followed one another. The recognition phase occurred twice, the second presentation reversing the order of the first.

Although Ss were asked to leave their earphones on for the duration of Experiment 1, these earphones were silent except for the presentation of instructions.

\section{RESULTS AND DISCUSSION}

The mean recognition rating for each type of new sentence (those appearing during recognition but not presented during acquisition) was calculated twice, once for each recognition trial. As Fig. 1 illustrates, the new sentences given the highest ratings on Trial 1 are the L-4s (2.71), followed by the L-3s (2.48), L-2s (2.37), and L-1s (1.12). A closely similar pattern was obtained for Trial 2 along with a general increase in misrecognition confidence for each type of sentence. The difference in recognition ratings for the four sentence levels was statistically significant, $F(2,14)=36.3, p<.01$. An a posteriori test on the mean recognition scores (Winer, 1962) indicated that it was the L-1 sentences that were different from the remaining sentences $(p<.01)$. The remaining sentence types did not differ significantly nor did performance vary from the first to the second recognition trial.

The pattern of scores illustrated in Fig. 1 is quite similar to those reported by Bransford and Franks (1971) and Cofer (1973, p. 540). These earlier investigators, however, employed a 10-point rating scale as opposed to the 5-point scale employed in the present study. The large scale led to greater differences between the sentences than those obtained with the 5-point scale. The general thrust of the findings, however, is once again to indicate that new complex sentences embodying the ideas of simpler sentences are more frequently misrecognized than are new simple sentences.

In order to determine whether different decision criteria were employed for the different sentence types, the procedures described by Green and Swets (1966) were used to calculate $\beta$ for each sentence type. The mean values of $\beta$ at the most cautious rating category are depicted in Fig. 2. For Trial 1, the mean value of $\beta$ was highest for L-1s (1.32) and decreased to .76 and .84 for $\mathrm{L}-2 \mathrm{~s}$ and L-3s, respectively. It was not possible to calculate $\beta$ for $L-4 s$, as these sentences occurred only during recognition. Essentially, the identical pattern of

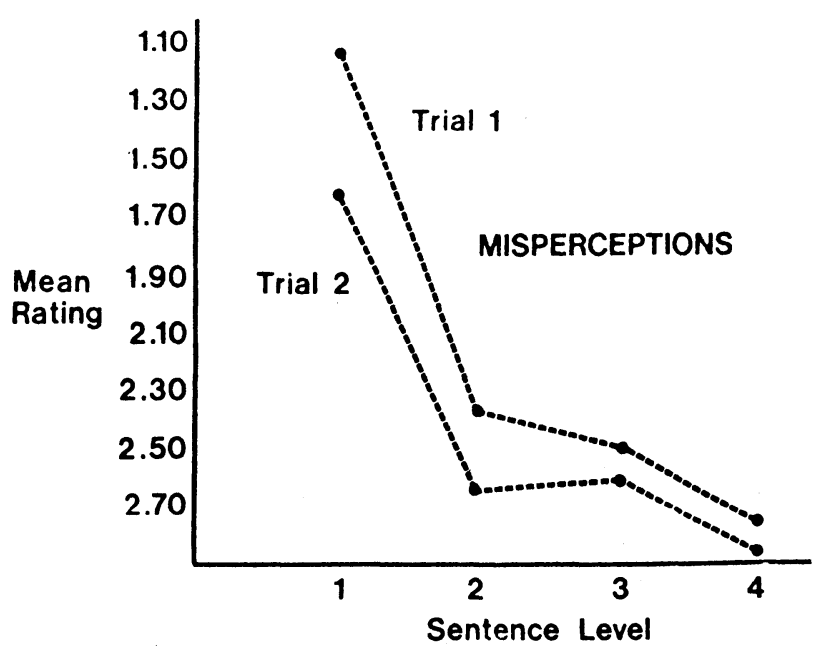

Fig. 1. Mean recognition rating for sentences presented only during recognition. 


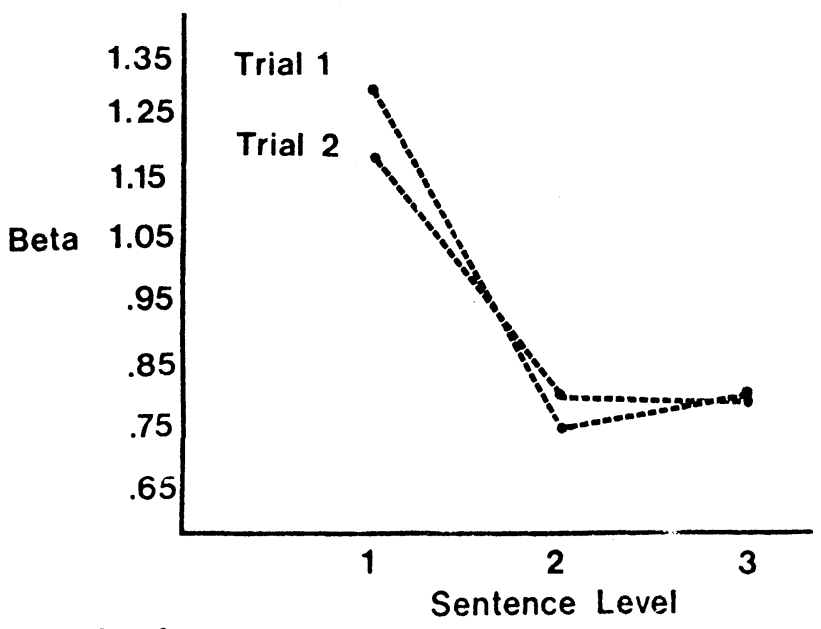

Fig. 2. $\beta$ for each sentence type. (Each point represents the mean $\beta$ for all of the sentences at that level.)

values was obtained on recognition Trial 2. These differences in $\beta$ were statistically significant, $F(2,14)=$ $33.10, \mathrm{p}<.01$. Once again, however, an a posteriori test indicated that while L-1 sentences were different from the others $(p<.01)$, the L-2 and L-3 sentences did not differ significantly nor did performance from Trial 1 to Trial 2. From these results there seems good reason to conclude that Ss employed a riskier criterion in deciding whether L-2 and L-3 sentences were "old" than in deciding whether L-1s were old.

In order to determine whether L-1 sentences were more easily remembered, $d^{\prime}$ was calculated for each sentence type at the most cautious rating category. Figure 3 illustrates these data and reflects, once again, the difference between L-1 sentences and the remaining sentences. Clearly, on Trial $1 \mathrm{~L}-1$ sentences were more easily remembered $\left(d^{\prime}=1.29\right)$ than either $L-2\left(d^{\prime}=.63\right)$ or L-3 $\left(\mathrm{d}^{\prime}=.39\right)$ sentences. A similar pattern was obtained for Trial $2, F(2,14)=31.57, \mathrm{p}<.01$, and, once again, the difference was due to the difference between the L-1 sentences and the others $(\mathrm{p}<.01)$. On Trial $2 \mathrm{~d}^{\prime}$ was generally lower for L-2s and L-3s but not L-1s. The interaction between sentence type and trials, however, was not statistically significant.

The results provide some support for the view that complex sentences expressing many of the ideas contained in simpler sentences are responded to with less caution than simple sentences in a recognition situation. The Ss used a less stringent criterion in making decisions about L-2 and L-3 sentences than they used for L-1 sentences. Unfortunately, it is not possible to conclude that the differences in misperception ratings were solely the results of schematic organization because memory for sentence form $\left(d^{\prime}\right)$ also differed. L-1 sentences were better remembered than either L- 2 or L-3 sentences. It is

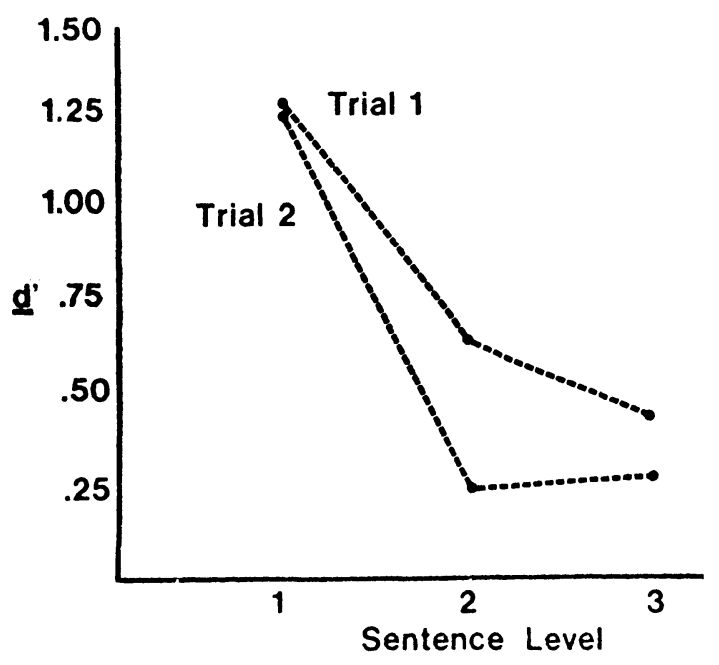

Fig. 3. $d^{\prime}$ for each sentence type. (Each point represents the mean $d^{\prime}$ for the sentences at that level.)

very likely, therefore, that L-1 sentences were rarely misperceived, at least in part, because their form was not as easily confused with new sentences as the poorly remembered L-2s and L-3s. The ability to retain the form of verbal materials for relatively long periods of time is in agreement with data presented by Kirsner (1973) and Kolers (1973), and even Cofer (1973) found form to play a role early in the recognition task. (Form, in the present experiment, also became somewhat less important with time.)

Finally, there is some evidence that retaining the form of a sentence is more important in the recognition of sentences with a large number of "abstract" words than in the recognition of "concrete" sentences (Paivio, 1971). The results of the present study indicate that form is also relied upon when other features of the task (the similarity of sentence meanings in the present case) demand it.

\section{REFERENCES}

Bartlett, F. C. Remembering. Cambridge, England: Cambridge University Press, 1932.

Bransford, J. D., \& Franks, J. J. The abstraction of linguistic id eas. Cognitive Psychology, 1971, 2, 331-350.

Cofer, C. N. Constructive processes in memory. American Scientist, $1973,61,537-543$.

Green, D. M., \& Swets, J. A. Signal detection theory and psychophysics. New York: Wiley, 1966.

Kirsner, K. An analysis of the visual component in recognition memory for verbal stimuli. Memory \& Cognition, 1973, 1, 449-453.

Kolers, P, A. Remembering operations. Memory \& Cognition, 1973, 1, 347-355.

Paivio, A. Imagery and verbal processes. New York: Holt, Rinehart \& Winston, 1971.

Winer, B. J. Statistical principles in experimental design. New York: McGraw-Hill, 1967.

(R eceived for publication May 13, 1974.) 